OPEN ACCESS

Edited by: Alfredo Addeo,

Geneva University Hospitals (HUG), Switzerland

Reviewed by:

Jin-Yuan Shih,

National Taiwan University, Taiwan

Bhumsuk Keam,

Seoul National University Hospital,

South Korea

*Correspondence:

Xing Niu

xniu@cmu.edu.cn

Specialty section:

This article was submitted to

Thoracic Oncology,

a section of the journal

Frontiers in Oncology

Received: 11 July 2019

Accepted: 25 September 2019

Published: 11 October 2019

Citation:

Zhu X, Chen L, Liu L and Niu X (2019) EMT-Mediated Acquired EGFR-TKI

Resistance in NSCLC: Mechanisms and Strategies. Front. Oncol. 9:1044.

doi: 10.3389/fonc.2019.01044

\section{EMT-Mediated Acquired EGFR-TKI Resistance in NSCLC: Mechanisms and Strategies}

\author{
Xuan Zhu ${ }^{1,2}$, Lijie Chen ${ }^{3}$, Ling Liu ${ }^{4}$ and Xing Niu ${ }^{5 *}$ \\ ${ }^{1}$ Institute of Translational Medicine, China Medical University, Shenyang, China, ${ }^{2}$ Department of Surgery, First Affiliated \\ Hospital of China Medical University, Shenyang, China, ${ }^{3}$ Department of Third Clinical College, China Medical University, \\ Shenyang, China, ${ }^{4}$ Department of College of Stomatology, China Medical University, Shenyang, China, ${ }^{5}$ Department of \\ Second Clinical College, Shengjing Hospital Affiliated to China Medical University, Shenyang, China
}

Acquired resistance inevitably limits the curative effects of epidermal growth factor receptor tyrosine kinase inhibitors (EGFR-TKIs), which represent the classical paradigm of molecular-targeted therapies in non-small-cell lung cancer (NSCLC). How to break such a bottleneck becomes a pressing problem in cancer treatment. The epithelial-mesenchymal transition (EMT) is a dynamic process that governs biological changes in various aspects of malignancies, notably drug resistance. Progress in delineating the nature of this process offers an opportunity to develop clinical therapeutics to tackle resistance toward anticancer agents. Herein, we seek to provide a framework for the mechanistic underpinnings on the EMT-mediated acquisition of EGFR-TKI resistance, with a focus on NSCLC, and raise the question of what therapeutic strategies along this line should be pursued to optimize the efficacy in clinical practice.

\section{Keywords: epithelial-mesenchymal transition, acquired resistance, therapeutic strategies, non-small-cell lung} cancer, epidermal growth factor receptor tyrosine kinase inhibitors

\section{INTRODUCTION}

Lung cancer is the leading cause of cancer mortality among males worldwide and females in more developed countries (1). Non-small-cell lung cancer (NSCLC) accounts for $85 \%$ of cases (2). Treatment with epidermal growth factor receptor tyrosine kinase inhibitors (EGFR-TKIs) has been the first-line treatment for NSCLC patients harboring activating EGFR mutations $(3,4)$. Nonetheless, an overwhelming majority of patients who initially respond to EGFR-TKIs treatment eventually develop acquired resistance, which invariably limits the clinical efficacy of therapy. Epithelial-mesenchymal transition (EMT) has long been linked to acquired EGFR-TKI resistance in NSCLC, but mechanisms underlying EMT-dependent acquisition of EGFR-TKIs resistance are still far from fully explored. EMT, a reversible biological process, wherein cells undergo a switch from epithelial phenotype to mesenchymal state. EMT is involved in both physiological and pathological processes. Originally described in the context of embryonic development, to date, EMT has been correlated closely with tumorigenesis, invasion, metastasis and drug resistance (5-7). Due to the reversibility of EMT process, further understanding of the intricate relationship between EMT and NSCLC, particularly the mechanistic basis responsible for EMT-mediated resistance contributes to improving the benefit of TKI treatment for NSCLC patients. In this review, we focus on potential mechanisms of acquired EGFR-TKI resistance induced by EMT and discuss promising avenues for targeting the EMT program as a strategy for NSCLC treatment. 


\section{EMT AND DRUG RESISTANCE}

EMT is a process involved profound changes in both morphology and physiology (6). During EMT, cells downregulate the expression of epithelial proteins containing E-cadherin, whereas upregulate the expression of mesenchymal proteins, such as $\mathrm{N}$-cadherin and vimentin. In addition, cells undergoing EMT are characterized by the loss of apico-basal polarity and intact cell-cell junctions, while acquisition of front-rear polarity and dramatic remodeling of the cytoskeleton (8). Also, EMT program endows cells with enhanced invasive capacity, therapeutic resistance and cancer stem-cell-like properties $(7,9)$. These findings have inspired the interests in EMT in the cancer field during the last decade. Resistance to EGFR-TKI represents a prime obstacle for NSCLC treatment, making it of particular importance to delve into the detailed mechanisms. Hence, how the execution of EMT contributes to drug resistance has been studied extensively.

\section{EMT Phenotype Attributes in EGFR-TKI Resistant NSCLC Cells}

A growing number of studies point to a molecular association between EMT and drug resistance. Some clinical and molecular evidences were established in the early 2010s. A lung cancer patient who developed acquired resistance to erlotinib was reported to be found EMT in the tissue sample. It is worth noting that there were no other known resistant mechanisms including T790M mutation and MET amplification. In an additional experiment, the gefitinib-resistant subline of HCC827 cells shows phenotypic and molecular changes that are consistent with EMT (10). Emerging evidence agrees that gefitinibresistance PC9 and HCC827 cells convert to a mesenchymal phenotype. Accompanied by the decreased expression of Ecadherin, the expression of $\mathrm{N}$-cadherin and other mesenchymal markers is elevated, illustrating the emergency of EMT (11). Furthermore, NSCLC cells with acquired resistance to gefitinib or osimertinib (AZD9291) showed EMT characteristics, with a decrease in E-cadherin, and increases in vimentin and stemness, without any EGFR secondary mutations (12). Taken together, it is not surprising that EMT is considered as one of the possible mechanisms for the acquired resistance to EGFR-TKIs in NSCLC.

\footnotetext{
Abbreviations: EGFR-TKIs, epidermal growth factor receptor tyrosine kinase inhibitors; NSCLC, non-small-cell lung cancer; EMT, epithelial-mesenchymal transition; siRNA, small interfering RNA; ZEB, zinc-finger E-box-binding; TGF- $\beta$, transforming growth factor- $\beta$; Grb2, growth factor receptor-bound2; HDAC6, histone deacetylase6; NICD, Notch intracellular domain; IGF, insulin-like growth factor; IGF-1R, insulin-like growth factor-1 receptor; IRS-1, insulin receptor substrate-1; MAML, Mastermind-like protein; Hh, Hedgehog; SHH, Sonic hedgehog; IHH, Indian hedgehog; DHH, Desert hedgehog; SMO, Smoothened; CSE, cigarette smoking extract; NAC, N-acetylcysteine; STAT3, signal transducer and activator of transcription 3; OSM, oncostatin-M; TEM, tumor microenvironment; JAK1, Janus Kinase1; miRNAs, microRNAs; 3'-UTR, $3^{\prime}$-untranslated region; PTEN, phosphatase and tensin homolog; CSCs, Cancer stem-like cells; SMIs, Small molecule inhibitors; FHD, first-in-human dose; mAbs, monoclonal antibodies; SAEs, serious adverse events; OS, overall survival; MTD, maximum tolerated dose; AEs, adverse events; mTOR, mammalian target of rapamycin; ASO, antisense oligonucleotide.
}

\section{Role of EMT in Resistance to Targeted Therapy}

Accumulating evidence has highlighted that activation of Notch signaling participates in EMT in NSCLC (13). Earlier studies discovered that aberrant Notch-1 signaling leads to acquired resistance to EGFR-TKI by triggering EMT and silencing of Notch-1 using small interfering RNA (siRNA) increases the sensitivity of gefitinib (14). The orchestrated changes in gene expression that favors EMT results from acting cooperatively of various master regulators, most notably Snail, Slug, Twist and zinc-finger E-box-binding (ZEB) transcription factors (8). Recent data have proven that the overexpression of Slug and Snail, which are identified as EMT inducers, promotes gefitinib resistance. Remarkably, recovery of TKI sensitivity is associated with the EMT reversion, since blocking the reversal of EMT by the forced expression of EMT inducers can suppress this effect (11). Collectively, these experimental observations reveal the notion that EMT is a vital event in the development of acquired resistance to EGFR-TKIs.

\section{Mechanisms Associated With EGFR-TKI Resistance via EMT}

Despite the vast body of studies regarding the role of EMT in targeted therapy for NSCLC patients, its potential mechanisms are not entirely clear. The mechanisms that govern the EMT are non-linear complex networks (15). Various extracellular signal factor stimuli and the activation of the corresponding intracellular signaling pathway, ultimately results in the downregulation of E-cadherin, which is considered as the hallmark of EMT (8).

\section{TGF- $\beta$ : Inducer of EMT}

Among flexible regulatory networks of EMT, one of the bestcharacterized inducers is transforming growth factor- $\beta$ (TGF$\beta$ ), which has context-dependent effects on cancer progression. TGF- $\beta$ can be tumor suppressive in pre-malignant epithelial cells by cell cycle arrest and oncogene suppression. But in the context of advanced carcinoma cells, the status of TGF- $\beta$ is assigned to pro-tumorigenic via mechanisms including promoting tumor angiogenesis, restraining the function of immune system as well as the activation of EMT $(16,17)$. Acting as a predominant inducer, TGF- $\beta$ can induce EMT through SMAD-medicated and non-SMAD pathways (Figure 1).

Based on data in cellular and animal models, exposure to gefitinib with increasing concentrations is sufficient to raise the level of TGF- $\beta$ secretion in HCC4006 and HCC827, going with increased phosphorylation of downstream proteins, SMAD2 and SMAD3. A TGF- $\beta$ autocrine loop is established by the EGFR inhibition in NSCLC with EGFR mutations, facilitating TGF- $\beta$-stimulated activation of SMAD pathway, thus playing a crucial role in EMT (18). How SMAD pathway contributes mechanistically to EMT has been documented. TGF- $\beta$ family receptor is a heterotetrameric complex with two type I and two type II components, comprising of three distinct regions: an extracellular $\mathrm{N}$-terminal ligand-binding domain, an intracellular C-terminal Ser/Thr kinase domain 


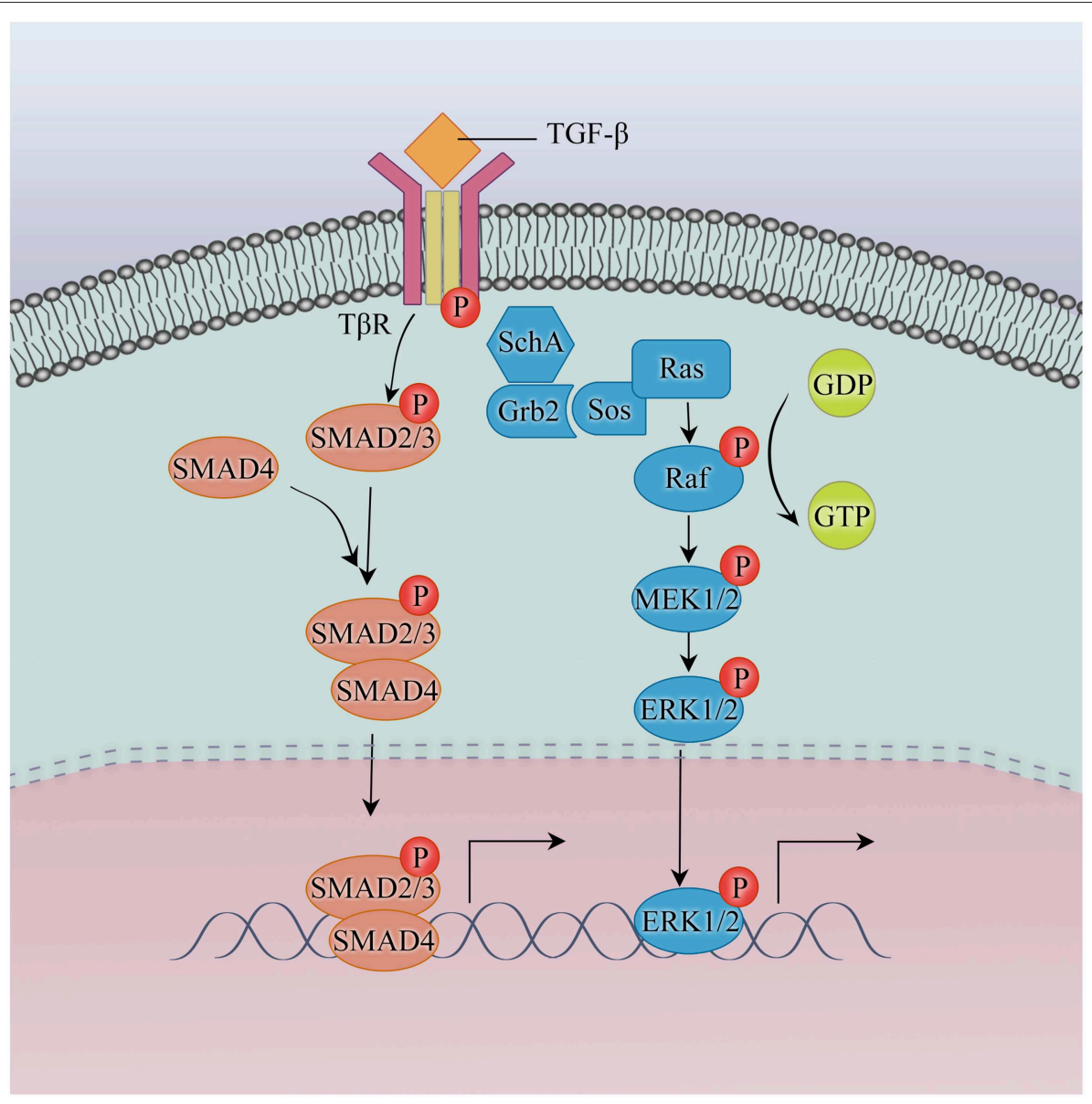

FIGURE 1 | TGF- $\beta /$ SMAD and non-SMAD pathways in EMT.

and centrally located transmembrane domain. Coupling of a ligand with TGF- $\beta$ receptor complexes, the T $\beta$ RII (type II) is activated through phosphorylation and thus activates the T $\beta R I$ (type I), which in turn phosphorylates the SMADs of kinase domain at the C-terminal sequence. In response to receptorphosphorylated SMAD2 and SMAD3 combination with SMAD4, the trimeric complexes subsequently translocate from cytoplasm to nucleus. Via interacting with the DNA-binding transcription factor, SMAD complexes serve as transcriptional coactivators to activate transcription of mesenchymal genes and transcriptional corepressors to repress transcription of epithelial genes (8).

SMADs also increase the activity of EMT transcription factors as well. ZEB1 is overexpression in HCC4006ER cells which take on mesenchymal characteristics and develop erlotinib resistance by the activation of TGF- $\beta /$ SMAD pathway. Correspondingly, knockdown of ZEB1 can reverse EMT and restore the sensitivity to TKI. This finding identifies ZEB1 as a remarkable regulator in TGF- $\beta /$ SMAD-related EMT and TKI resistance (19).

Apart from its role through SMAD pathway, TGF- $\beta$ activates the MAPK pathway, which also results in EMT-mediated resistance to EGFR-TKI. Blocking ERK signaling by MEK1/2 inhibitor can prevent TGF- $\beta$-induced EMT, indicating that MAPK pathway plays a key role in the induction of EMT by TGF- $\beta$ (5). Upon TGF- $\beta$ stimulation, the tyrosine and serine phosphorylation of SRC homology 2 domain-containingtransforming A (SchA) is induced, which is mechanistically associated with T $\beta$ RI. Phosphorylated SchA possesses a growth factor receptor-bound-2 (Grb2) binding site and induces its association with son-of-sevenless (Sos), then converts Ras into active Ras-GTP, thereby initiating the downstream Ras-RafMEK1/2-ERK1/2 cascade (20). Of note, enhanced sensitivity to gefitinib is in line with changes in epithelial and mesenchymal markers. After reaching a peak, heightened responses to gefitinib by chronic MEK inhibition eventually decline (5). In light of this, discovering alternative signaling inhibitions that render more stable maintenance of an epithelial phenotype awaits further investigation.

Additionally, crosstalk exists between the TGF- $\beta$ and Notch signaling to coordinately regulate the reprogramming of gene expression in EMT (21). TGF- $\beta$ can significantly increase the expression of Notch downstream target genes, namely HEY1 and HES-1. Consistent with this notion, pharmacological inhibition of Notch by histone deacetylase6 (HDAC6)-targeted siRNA leads to decreased expression of EMT genes, HEY1 and HES-1, suggesting that HDAC6 is required for Notch activation by TGF- $\beta$. Further experiment illuminates HDAC6 


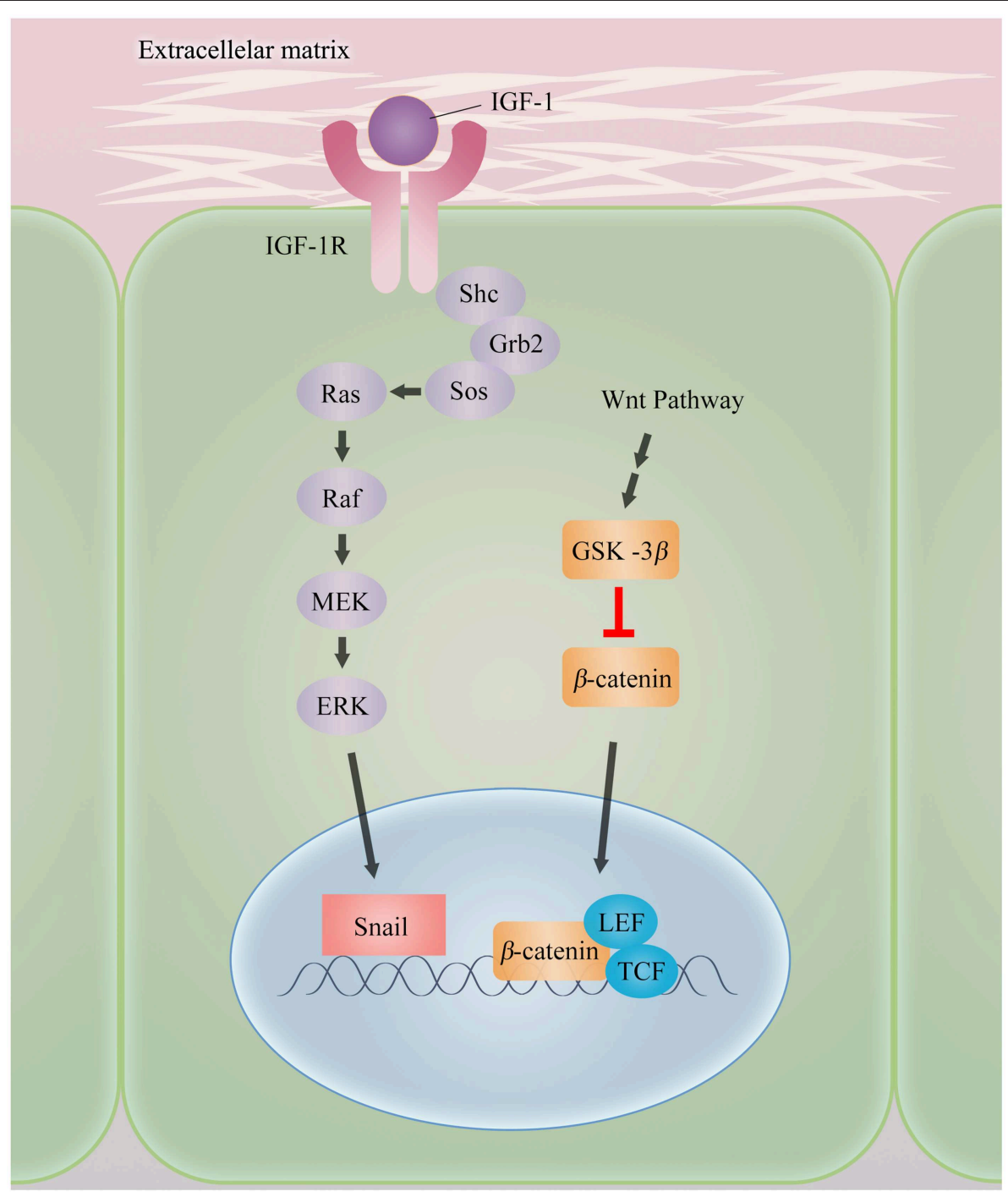

FIGURE 2 | Mechanisms of IGF-1R-induced EMT.

is activated then deacetylates its substrate, HSP90, in response to TGF- $\beta$ stimulation, which is of necessity in the cleavage of Notch receptor. This enables the release of Notch intracellular domain (NICD) and then enter the nucleus, where in cooperation with RBP-J (also known as CSL) as a transcription cofactor to regulate the expression of target genes, including Hey and Hes (22). Given that overexpression of HDAC6 is common in lung adenocarcinoma cell lines and confers resistance to gefitinib, interplay amongst TGF- $\beta$, Notch signaling and HDAC6 may be implicated in EMT and resistance to EGFR-TKI (23).

\section{Induction of IGF1 and EMT}

The insulin-like growth factor (IGF) signaling is thought to play a particularly prominent role in growth, development and apoptosis. The insulin-like growth factor-1 receptor (IGF-1R), a key signaling element in the IGF system, is a transmembrane tyrosine kinase receptor, which exerts its effect by binding to the ligand IGF-1. Once activated, IGF-1R is capable of intrinsic tyrosine kinase phosphorylation and activating multiple intracellular adaptor proteins, such as insulin receptor substrate1 (IRS-1) and Shc to transmit signals, leading to the activation of downstream signaling pathway $(24,25)$. Recently, various studies have revealed that IGF-1/IGF-1R also appears to take part in EMT and drug resistance $(24,26,27)$. NSCLC patients with elevated expression of IGF-1R show poor responses to EGFRTKIs treatment, strongly suggesting that activation of the IGF$1 \mathrm{R}$ pathway is relevant to resistance against EGFR-TKIs (27). However, the importance of IGF-1R-induced EMT in driving the resistance to TKI, specially NSCLC, remains largely obscure.

Several works have been undertaken to elucidate the interplay between IGF-1R and TKI resistance (Figure 2). For example, in a model of EGFR-TKI-resistant NSCLC, in which along with IGF-1R upregulation, cells display a highly EMT phenotype. Furthermore, silencing of IGF-1R or overexpression 


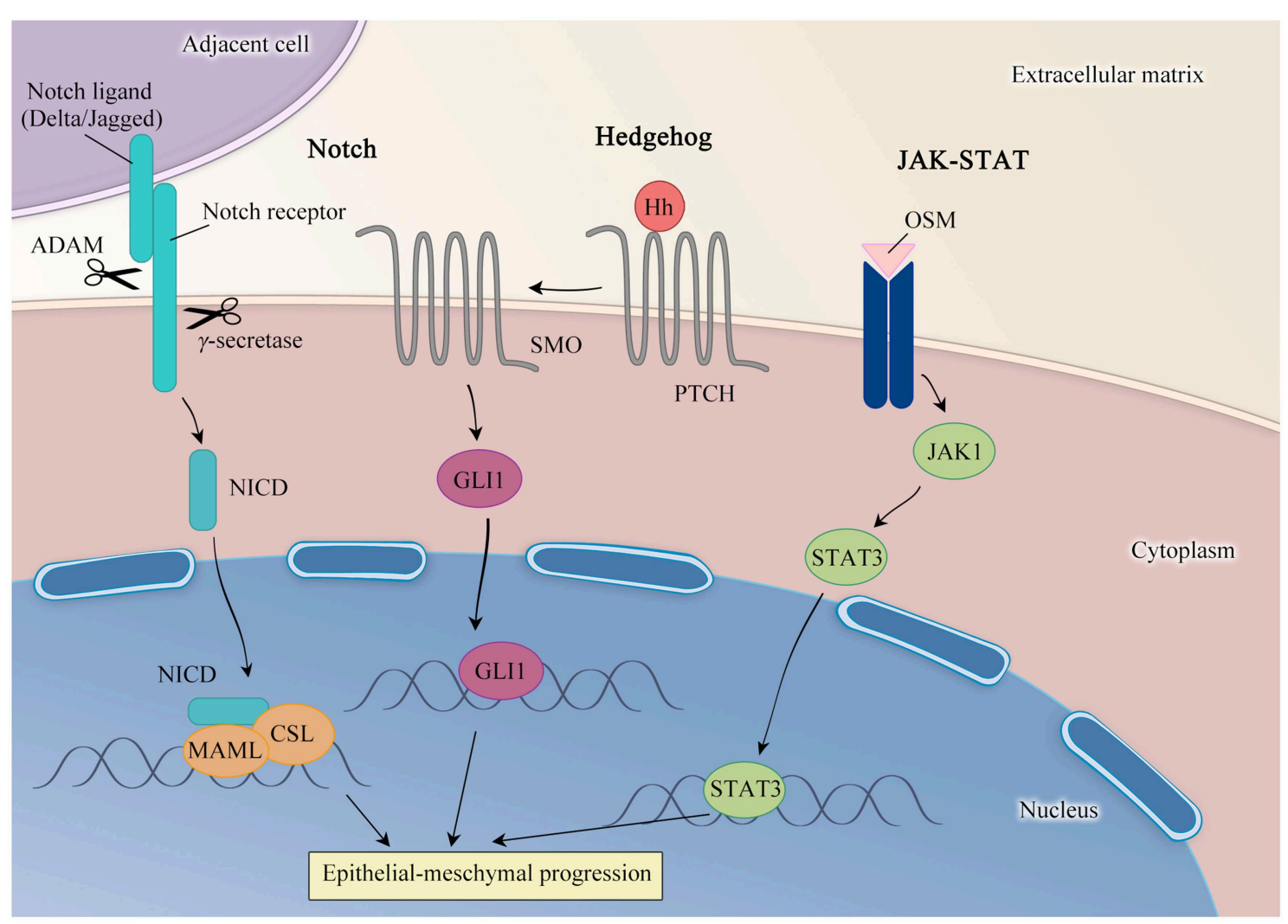

FIGURE 3 | Key signaling pathways involved in EMT.

of E-cadherin dramatically represses the EMT program and decreases the cells survival against EGFR-TKI. It should be noted that the expression of Snail and nucleus $\beta$-catenin are subsequently enhanced in IGF-1R-dependent EMT. In addition, not PI3k/AKT pathway, but rather the activation of ERK/MAPK signaling is proven to contribute critically to this process in both PC-9 and HC460 cell lines (26). Taken as a whole, these experimental observations provide direct evidence that upregulating of Snail expression arising from activating ERK/MAPK signaling and propelling $\beta$-catenin relocation from the cell membrane into nucleus might engender IGF-1R-induced EMT in NSCLC. The synergistic cooperation between these two processes stifles the expression of E-cadherin directly, thereby conferring mesenchymal attributes and acquired resistance to EGFR-TKIs in NSCLC. Of interest, $\beta$-catenin is the downstream signal molecule of Wnt signaling. Wnt signaling is considered to promote EMT by impeding the function of glycogen synthase kinase-3 $\beta$ (GSK-3 $\beta$ ) to stabilize $\beta$-catenin, which translocates to the nucleus and interacts with the transcription factors lymphoid enhancer-binding factor 1 (LEF) and T cell factor (TCF) to define EMT (8). It is increasingly clear that the presence of interaction between the IGF-1R and Wnt/ $\beta$-catenin in the context of EMTmediated TKI resistance.

In contrast to the prevailing studies that focus on the impact of specific receptors in resistance, a report has furnished evidence from different viewpoints. Genetic deletion is used to functionally remove IGF-1R in HCC827 cells. Upon exposure to continuous high-dose erlotinib, HCC827 (IGF-1R ${ }^{-/-}$) ER cells do not exhibit an EMT phenotype shift like HCC827ER cells as expected. Intriguingly, acquired resistance is developed by bypass signaling, namely MET-amplification, indicating that IGF-1R is not essential to maintain the gained TKI-resistant state in NSCLC (28). Indeed, results of these data offer us better insights into the resistance by induction of IGF-1R and underscore the complexity of underlying resistance mechanisms, that is under a given TKI selective pressure, cells are prone to find another way to develop resistance in the absence of certain mechanism.

\section{Key Signaling Pathways in EMT}

As with growth factors mentioned above, Notch signaling has been recognized as a potent mediator of EMT (Figure 3). In mammals, there are four receptors (Notch1-Notch4) and two families of Notch ligands, Delta-like (DLL1/3/4) and Jagged-like (JAG1/2) have been identified. Simply speaking, Notch signaling is an intercellular communication mechanism, in which a Notch ligand on the surface of cell induces a suite of proteolytic events including sequential cleavage by ADAM enzymes and then $\gamma$ secretase. These events permit the liberation of NICD, which translocates to the nucleus to modulate the expression of Notch target genes in company with CSL (also known as RBP-J) and Mastermind-like protein (MAML) as a result $(29,30)$. 
In a previous experiment, inappropriate activation of Notch1 inhibit gefitinib-induced apoptosis relying on caspase3 inactivation caused by Notch1-induced EMT, Accordingly, out of balance between apoptosis and anti-apoptosis allows resistance to EGFR-TKI (14). Recently, studies in cell culture have exemplified that chronic treatment of gefitinib dramatically enhances ZEB1 expression, together with the repression of miR200c. Meanwhile, an EMT state is observed in gefitinib-resistant cells. Conversely, knockdown of Notch-1 enables partially reverse EMT and gefitinib resistance, sharing similarity with the effect of miR-200c. This result implies that Notch1 facilitates EMT to mediate ZEB1-induced TKI resistance. Furthermore, treatment with the $\gamma$-secretase inhibitor to block Notch signaling, cells gain growing sensitivity to gefitinib, manifesting the central role of Notch in EGFR-TKI resistance and promising therapeutic value for NSCLC patients with EMT-induced resistance. Nevertheless, consideration of both necessary and sufficient part of NICD to transcriptionally attenuate ERBB3 expression by binding to its promoter directly, inhibition of Notch1 inevitably induces ERBB3, a driver of EGFR-mutated lung cancer cell growth (31). To summarize, these findings support the versatile role of Notch1 in EGFR-mutated cells. Under the circumstance that EGFR is inhibited by TKI treatment, Notch1 results in the acquisition of resistance by triggering EMT. Of particular note, the fact that Notch1-ERBB3 axis functions as a universal regulatory mechanism in NSCLC, provides solid evidence and attractive possibilities for combined targeting of Notch1 and EGFR to treat EMT-driven resistance to EGFR-TKI (31). This result, as well as the prior one, validate the knowledge that Notch signaling is a robust contributor of EMT-mediated TKI resistance.

Outside of Notch cascade, the Hedgehog ( $\mathrm{Hh}$ ) pathway is frequently linked with EMT and dwindling response to EGFRTKI treatment. There are three $\mathrm{HH}$ counterparts have been made clear in human: Sonic hedgehog ( $\mathrm{SHH}$ ), Indian hedgehog $(\mathrm{IHH})$ and Desert hedgehog $(\mathrm{DHH})$. In the absence of $\mathrm{HH}$ binding, Patch (PTCH) suppresses the activity of Smoothened (SMO), a member of the $\mathrm{G}$ protein-coupled receptor superfamily. Interaction of $\mathrm{HH}$ ligands with $\mathrm{PTCH}$ initiates the $\mathrm{Hh}$ signaling cascade through the relief of its inhibitory effect on SMO, giving rise to activation of GLI1, a zinc-finger transcription factor, to play its part in the nucleus $(32,33)$.

Mounting evidence demonstrates that dysregulation of this pathway is crucial for EMT-dependent resistance. In vitro model of acquired resistance to EGFR-TKIs indicates that HCC827GR cells present a mesenchymal signature accompanied by markedly elevated expression of GLI1 and SMO amplification, implying an involvement of the $\mathrm{Hh}$ pathway in the course of EMT. Strikingly, interplay between $\mathrm{Hh}$ and MET is described in cell lines and tumor xenografts of nude mice. Depletion of SMO and MET concomitantly enhances gefitinib sensitivity and significantly diminishes the phosphorylation level of MAPK and AKT proteins. In brief, excessive activation of $\mathrm{Hh}$ pathway, drives EGFR-TKI resistance owing to EMT induction via SMO amplification and concurrent MET activation. Therefore, the combination of $\mathrm{Hh}$ and MET inhibitors may yield powerful antitumor effects in EGFR-mutated NSCLC patients (34).
More recently, further cue to emerging importance of $\mathrm{Hh}$ signaling in the induction of EMT derives from a series of studies. For instance, findings of a research show the functional links among Hh signaling, EMT, CSC abundance to the acquired resistance of EGFR-TKI (35). Additional experiment designed to investigate the efficacy and mechanisms of acquired resistance during the sequential treatment with first-, second- and thirdgeneration EGFR-TKIs reveals that activation of the Hh pathway is a common nature that all of three lines share. Different from the preceding result in a model of the first-generation inhibitor, MET hyperactivity is not detected in second- and third-generation resistant models, while SMO activation coexists persistently in diverse treatment (36). In addition, synergic role of AXL and Hh pathway has been reported to mediate resistance to second- and third-generation EGFR-TKIs (36). Given all above, the idea that Hedgehog pathway behaves as a fundamental player for EMTmediated acquired EGFR-TKIs resistance is little surprising.

Other factors are noted to aid the induction of EMT and cancer progression. Src has been reported to elicit cigarette smoking extract (CSE)-induced EMT and resistance to gefitinib, while $\mathrm{N}$-acetylcysteine (NAC) abrogates the resistance through alleviating Src activation and EMT, providing a clue that simultaneous targeting of EGFR-TKI and Src may help in clinical outcomes in EGFR-mutated NSCLC patients with smoking history (37). Another report indicates that smoking abolishes EGFR-TKI therapeutic effects in NSCLC on account of continuously activating ERK1/2 and AKT pathway downstream of EGFR signaling as well as EMT induction (38). Besides, studies have shown that metformin, a well-known antidiabetic drug, effectively overcomes resistance to EGFR-TKI in vitro and in vivo, by reversing EMT and suppression of interleukin (IL)6/signal transducer and activator of transcription 3 (STAT3) pathway (39). Recent advance reveals that anticancer drugs activate IL-6 proinflammatory pathway with upregulation of IL6 and oncostatin-M (OSM) expression. It is noteworthy that acquired resistance cell lines display genic and morphologic changes, suggesting the occurrence of EMT. In addition to STAT3 activation by autocrine, cocultured with cancer-associated fibroblasts (CAFs) in NSCLC cells in culture also gives rise to inflammation in the tumor microenvironment (TEM) via the secretion of IL-6 and OSM, thus leading to resistance to TKIs. However, this result can be prevented by Janus Kinase1 (JAK1) knockdown. In this context, OSMRs/JAK1/STAT3 axis has been proposed to result in TKI resistance in NSCLC (40).

\section{Regulation of miRNA on EMT}

Beyond regulatory networks at the transcriptional level, EMT program is tightly controlled by microRNAs (miRNAs), which has been brought in to focus currently. MiRNAs, small noncoding single-stranded RNAs encompassing 19-25 nucleotides, that modulate gene expression post-transcriptionally, are found to exert pivotal impacts on a variety of biological processes in the development of cancers (41-45). Through coupling with complementary sequences via incompletely base-pairing in the $3^{\prime}$-untranslated region ( $3^{\prime}$-UTR), miRNAs are able to silence EMT-related molecules, which favor or repress the progress of EMT (Figure 4) (44). 


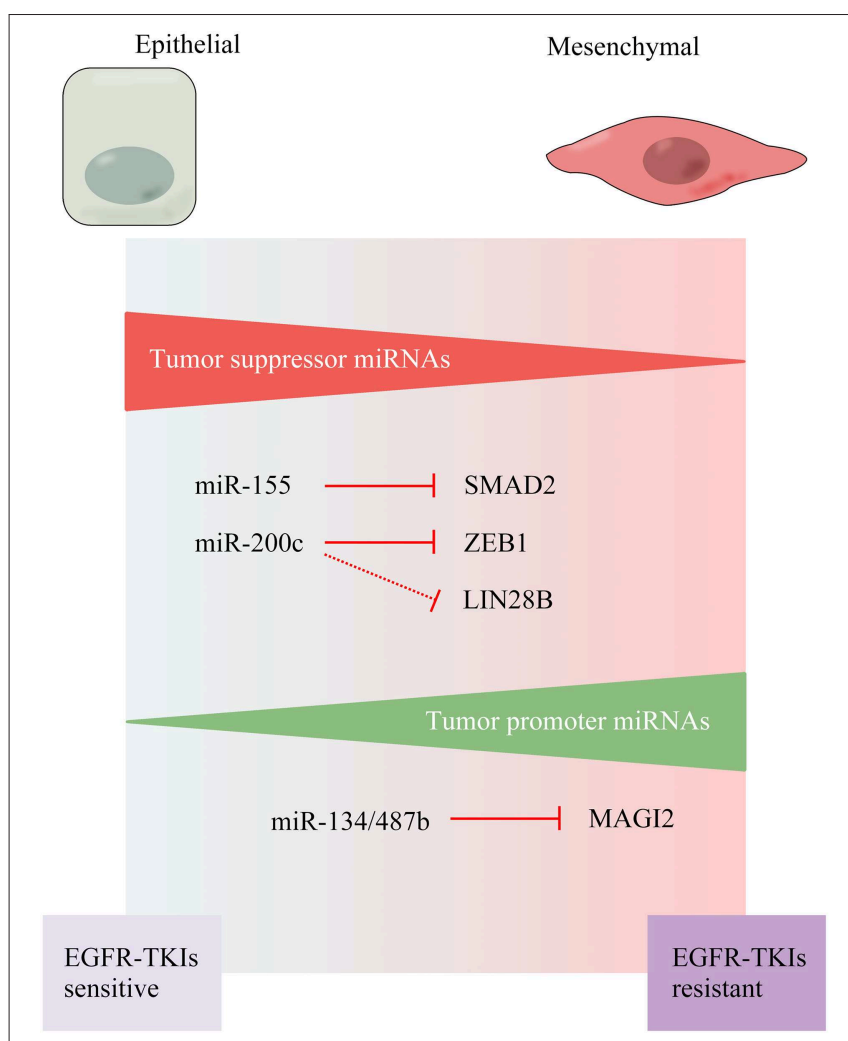

FIGURE 4 | miRNAs regulating EMT program and susceptibility to targeted therapy.

Several lines of evidence shed some light on how the resistance to EGFR-TKI is generated by miRNAs regulation. Experiments in cell lines of NSCLC illuminate that long-term treatment with gefitinib renders changes in miRNAs expression, including depletion of miR-155 and miR-200c. Furthermore, together with the augmented protein level of SMAD2 and ZEB1, the expression of E-cadherin is sharply declined, at least in part, depending on histone modification. In support of this idea, under co-treatment of miR-155 and miR-200c inhibitors, similar results are shown in terms of protein expression and appearance of mesenchymal profile (46). As mentioned, miRNA200 family members, strong negative regulators of EMT, have drawn substantial attention in tumor biology (47). Analyses of NSCLC cell lines explain that epigenetic modifications, such as promoter hypermethylation, are accounting for the aberrant silencing of miR-200c. Moreover, acquired resistance NSCLC cells bearing the EMT feature exhibit low expression of miR200c and high LIN28B expression. On the other hand, miR-200c induction not only suppresses the expression of ZEB1, which is known as a mesenchymal marker, but also LIN28B in parallel. Alternatively, knockdown of LIN28B also poses an antitumor effect (48). Overall, the miR-200c/LIN28B axis is complicit in NSCLC resistant against EGFR-TKI. Identification of such a fundamental mechanism involving miR-200c and LIN28B is an ongoing topic of research to pave the way to curative remedy in future.
As is apparent from these instances, miR-200 family members have been depicted as primary miRNA suppressors. For another, tumor promoter miRNAs, provide an additional approach to induce EMT by inhibiting the molecules suppress this step or provoking those initiate it (44). Overexpression of miR134 and miR-487b as a consequence of TGF- $\beta$ stimulation has been portrayed to contribute to the occurrence of EMT and drug resistance in NSCLC. Compatible with this result, transfection of anti-miR134/487b inhibits the EMT phenomenon and regains sensitivity to gefitinib likewise. Membrane-associated guanylate kinase, WW, and PDZ domain-containing protein 2 (MAGI2), a scaffold protein is required for phosphatase and tensin homolog (PTEN) stability, is a direct target of miR134 and miR-487b. Reduction of MAGI2 caused by TGF- $\beta$ leads to PTEN phosphorylation and PI3k/AKT cascade activation. It is therefore promoting the TGF- $\beta$-induced EMT and acquired resistance to EGFR-TKI in NSCLC (48).

In summary, numerous studies on the regulation of EMT process by miRNAs unravel their functional roles as tumor suppressors or tumor promoters. MiRNAs seem to participate in carcinoma progression by targeting EMT-related mRNAs in elaborate regulatory networks at the post-transcriptional level, imbuing an additional layer of gene expression. Consequently, these miRNAs are likely to dictate how activation of signaling pathways affects cell fate and determinate the response to targeted agents. Despite the research advances concerning molecular mechanisms of miRNAs in the regulation of EMT in cancers, little is known about its relative contribution on EMT-mediated acquisition of EGFR-TKI resistance, especially in NSCLC. Future effort is needed to fill these gaps for improvements in therapy.

\section{Relationship Between EMT and CSC}

As discussed previously, the conversion of EMT is a key component in malignancy, resulting in disease progression and drug resistance. Cancer stem-like cells (CSCs) are another strong driving force in the development of therapeutic resistance, ranging from conventional chemotherapeutics to molecularly targeted therapy $(49,50)$. The CSC paradigm postulates the existence of a minor subpopulation of neoplastic cells that hold self-renewal ability to generate daughter CSCs and differentiation potency to produce non-CSCs progenies. Implicit in this paradigm is the capacity of seeding new tumors and the generation of tumor heterogeneity (51).

Currently available evidence points to the mutual relationship between these two phenotypes in EGFR-TKI resistance. One example is the identification of EMT features and stem celllike properties in a study based upon afatinib-resistant cell lines, revealing a possible association in the midst of EMT, stem cell-like signature and EGFR-TKI resistance (52). Data from a model with ectopic activation of Hh pathway, NSCLC cells exhibit EMT attributes and ABCG2 upregulation. ABCG2, have been referred as a stem cell marker, severs as multidrug resistance pumps to EGFR-TKIs, which is controlled by $\mathrm{Hh}$ signaling directly. Blocking Hh pathway by a specific inhibitor, NSCLC cells become able to resensitize to EGFR-TKI, along with the reversal of EMT phenotype and CSCs reduction (35). In addition, analyses of the molecular characteristics 
of gefitinib-resistance chemokine receptor 4 (CXCR4)-positive cells have unveiled that upregulation of CXCR4, to some degree, is induced by EMT. Given the knowledge that CXCR4-positive cells have the property of stemness, it is conceivable that CXCR4 is a hub to find the connection between EMT and CSCs, both of which correlate intimately with EGFR-TKI resistance (53). More recently, results of invitro assays and murine model, have reinforced the view that TKI treatment triggers EMT and confers a CSC phenotype in NSCLC cells, subsequently contributing to drug resistance. Further exploration reveals that the imperative character of AKT/FOXM1/stathmin axis in TKI-induced CSC enrichment and drug resistance, which is verified by the assessment of EMT and CSC biomarkers. Importantly, genetic manipulation of FOXM1 and stathmin 1, or blockade of PI3k/AKT pathway may impair CSC abundance and improve the response toward TKI agents (9).

Taking these into account, the connection between EMT and a CSC phenotype is proposed by massive experimental data, the mechanistic basis within these two events is unsolved yet. Since EMT appears to be a crucial strategy employed by tumor cells to acquire CSC-like properties, which are coupled to promote the potential of resisting to antitumor drugs, making EMT an appealing biological target for cancer therapy. Thus, a surge of attention has been given to targeting EMT regulators to eradicate CSCs (54). As such, whether EMT program is a necessary or sufficient condition for the enrichment of CSCs, and what is the relationship as well as the distinction between EMT and CSCs remains to be addressed. Given that CSCs are subjected to robust regulation by tumor microenvironment, one possible explanation is that alternations in diverse factors induced by carcinoma cells undergoing EMT change surrounding microenvironment, which affects the induction and maintenance of CSCs (55).

\section{STRATEGIES TO OVERCOME EMT-DEPENDENT ACQUISITION OF EGFR-TKI RESISTANCE}

Drug resistance is a pervasive barrier in TKI therapy. Unremitting studies enable us to better understand its biological underpinnings in lung cancer and lend added insights into clinical implications, such as novel therapeutic strategies to combat TKI resistance (56). EMT, a well-coordinated process, has been viewed as a major mechanism of EGFRTKI resistance in NSCLC, in this setting, strategies aimed at extracellular stimuli and intracellular signaling pathways related to EMT are rapidly accumulating (57). In this section, we recapitulate the emerging agents that impinging on EMTassociated signaling networks in the field of therapeutic intervention for NSCLC (Table 1).

\section{Targeting TGF- $\beta$}

TGF- $\beta$ can foster cancer progression by stimulating EMT, identification of its pro-oncogenic role has provided a rationale for the development of drugs targeting TGF- $\beta$ in cancer treatment. However, the pleiotropic functions of TGF- $\beta$ pose a formidable hurdle for the application of this strategy (58). Small molecule inhibitors (SMIs) embody a spectrum of pharmacological approaches to reversing EMT by blockage of TGF- $\beta$ signaling, among them galunisertib (LY2157299) is the most well-studied one (59). It had an acceptable safety profile and achieved success with a manner of intermittent dosing regimen based on the preclinical pharmacokinetic/pharmacodynamic models and predictive biomarkers development in a first-inhuman dose (FHD) study in glioma patients (60). Given the encouraging results of the FHD study, a nonrandomized, openlabel phase I clinical trial was conducted in Asian populations, 12 advanced solid tumors Japanese patients were treated with the improved dosage in lack of cardiotoxicity or other doselimiting toxicities during the treatment of galunisertib (61). Recently, a phase 2 study demonstrated that galunisertib plus sorafenib showed acceptable safety and a prolonged overall survival outcome for advanced hepatocellular carcinoma (62). Owing to cardiac toxicities in animals, the implementation of comprehensive cardiac monitoring throughout the course of the FHD study becomes an urgent imperative. Yet, administration of LY2157299 did not observe significant cardiac toxicity in either a short or long therapeutic procedure manner, thereby providing support for advancing clinical development (63).

Another avenue for blocking this pathway is immunotherapy. Belagenpumatucel-L, an allogeneic tumor cell vaccine, was evaluated in a randomized, placebo-controlled phase III study in III/IV NSCLC patients. Results in this work showed that it was well tolerated and rendered better survival compared to the placebo cohort. Unsatisfactorily, patients who completed the previous chemotherapy more than 12 weeks failed to receive any advantages. The clinical utility of belagenpumatucel- $\mathrm{L}$ merits further evaluation as a consequence (64).

\section{Targeting IGF-1R}

IGF-1R pathway also promotes EMT and evolution of resistance against TKI in clinical practice, hence IGF-1R pathway has evolved into an important target across different malignancies. Therapeutic agents studied in clinical trials involving patients with NSCLC include both monoclonal antibodies (mAbs) to IGF$1 \mathrm{R}$ and small molecule tyrosine kinase inhibitor of IGF-1R (65). Two phase III trials assessed the combination of figitumumab with cytotoxic chemotherapy (paclitaxel and carboplatin) and EGFR-TKI (erlotinib) in patients with advanced NSCLC were closed early with disappointing consequences $(66,67)$. The all-causality serious adverse events (SAEs) and treatmentrelated deaths in a subset of patients receiving figitumumab plus carboplatin/paclitaxel, as compared with those receiving chemotherapy alone, witnessed the failure of this clinical trial (67). Similarly, figitumumab combined with erlotinib did not hold superiority over erlotinib alone in another phase III study (66). Whereas these unimpressive outcomes in the clinic, further clinical development of figitumumab has been halted prematurely. However, in a recent phase II trial, chemotherapynaïve patients harboring activating EGFR mutations with stage IIIB/IV or post-surgical recurrent non-squamous NSCLC 
TABLE 1 | Overview of clinical data involving EMT-related pathway inhibition in NSCLC.

\begin{tabular}{lllll}
\hline $\begin{array}{l}\text { Mechanism of } \\
\text { drug action }\end{array}$ & Drugs & Phase & Tumor type & Preliminary results
\end{tabular}

TGF- $\beta$ signaling inhibitors

$\begin{array}{llll}\begin{array}{l}\text { TGF- } \beta \text { kinase } \\ \text { inhibitor }\end{array} & \begin{array}{l}\text { Galunisertib } \\ (\text { LY2157299) }\end{array} & \text { । } & \text { Solid tumors } \\ & \begin{array}{l}\text { Galunisertib } \\ \text { (LY2157299) }\end{array} & \text { I } & \begin{array}{l}\text { Glioma and solid } \\ \text { tumors }\end{array} \\ \text { Bumor cell vaccine } & \text { Belagenpumatucel-L } & \text { III } & \text { NSCLC }\end{array}$

\section{IGF-1R signaling inhibitors}

IGF-1R mAb

Figitumumab

Figitumumab

IGF-1R/INSR kinase

Linsitinib (OSI-906)

inhibitor

Linsitinib (OSI-906)

IGF-1R kinase

AXL1717

inhibitor

$$
\text { AXL1717 }
$$

RAS-RAF-MAPK inhibitors

$\begin{array}{ll}\text { BAF inhibitor + MEK } & \text { Dabrafenib + } \\ \text { inhibitor } & \text { Trametinib }\end{array}$

\section{PI3k-AKT-mTOR inhibitors}

mTOR inhibitor

Pictilisib (GDC-0941)

Everolimus

AKT inhibitor

MK-2206

\section{Notch signaling inhibitors}

\section{$\Gamma$-secretase \\ inhibitor (GSI)}

$\begin{array}{ll} & \text { RO4929097 } \\ & \text { BMS-906024 } \\ & \text { LY900009 } \\ \text { DLL4 mAb } & \text { Enoticumab } \\ & \text { (REGN421) } \\ & \text { Demcizumab }\end{array}$

Hedgehog signaling inhibitors

SMO inhibitor

PF-04449913
TAK-441
Sonidegib (LDE225)

\section{Other inhibitors}

IL-6 inhibitor

Siltuximab

\section{III}

III

॥

$\|$

I

$\|$

$\|$

NSCLC

Solid

tumors/NSCLC

NSCLC

Solid tumors

Solid tumors

Solid tumors

Preclinical

Leukemia and solid tumors

Advanced cancer

Solid tumors

NSCLC

Solid tumors

Solid tumors

Solid tumors
An acceptable tolerability and safety profile

No medically relevant cardiac toxicity detected

Good tolerability, no serious safety issues, no improvement in patients' survival after platinum-based chemotherapy

No improvement in patients' survival after figitumumab plus chemotherapy

No improvement in patients' survival after erlotinib plus figitumumab

A poor patients' prognosis caused by linsitinib plus erlotinib

No improvement in patients' survival after linsitinib plus erlotinib

Bone marrow toxicity profile

Low incidence of grade $3 / 4$ neutropenia

(61)

Clinically meaningful antitumour activity and a manageable safety profile

Well-toleration and preliminary antitumor activity

$(72-74)$

Measurable, dose-dependent, biologic, metabolic, and antitumor activity of everolimus in early-stage NSCLC

Well-toleration and preliminary antitumor activity for MK-2206 plus carboplatin and paclitaxel, docetaxel, or erlotinib

Well-toleration and a dose-dependent pharmacokinetic profile

Autoinduction at all dose levels that limited the ability to dose escalate the doses

$-$

Recommended maximum tolerated dose at 30 $\mathrm{mg} / \mathrm{kg}$ Q3W

Recommended phase II dose of $4 \mathrm{mg} / \mathrm{kg}$ Q $3 W$ and $3 \mathrm{mg} / \mathrm{kg}$ Q2W

Identification of a truncated dosing regimen and recommended phase II dose of demcizumab (5 mg/kg q3-weekly x4)

Maximum tolerated dose at $320 \mathrm{mg} /$ day, with preliminary antitumor activity

Maximum tolerated dose at 1,600 mg/day, with preliminary antitumor activity

Tolerance differences between East Asian and Western populations
(65)

(66)

(67) 
TABLE 1 | Continued

\begin{tabular}{|c|c|c|c|c|c|}
\hline $\begin{array}{l}\text { Mechanism of } \\
\text { drug action }\end{array}$ & Drugs & Phase & Tumor type & Preliminary results & References \\
\hline \multirow[t]{2}{*}{ STAT3 inhibitor } & AZD9150 & । & $\begin{array}{l}\text { Lymphoma and } \\
\text { lung cancer }\end{array}$ & $\begin{array}{l}\text { AZD9150 preclinical activity translated into } \\
\text { single-agent antitumor activity }\end{array}$ & (90) \\
\hline & OPB-51602 & 1 & Solid tumors & $\begin{array}{l}\text { A longer half-life and poorer tolerability of continuous } \\
\text { dosing compared with intermittent dosing }\end{array}$ & (91) \\
\hline \multirow[t]{2}{*}{ HDAC inhibitor } & Panobinostat (LBH589) & 1 & $\begin{array}{l}\text { NSCLC and } \\
\text { head-and-neck } \\
\text { cancer }\end{array}$ & $\begin{array}{l}\text { Maximum tolerated dose at } 30 \mathrm{mg} \text { (panobinostat) } \\
\text { and } 100 \mathrm{mg} \text { (erlotinib) }\end{array}$ & (95) \\
\hline & Romidepsin & I & NSCLC & $\begin{array}{l}\text { A well-tolerability and effects on relevant molecular } \\
\text { targets }\end{array}$ & (96) \\
\hline
\end{tabular}

were treated with erlotinib induction at $150 \mathrm{mg} /$ day for 3 months, followed by cytotoxic chemotherapy with platinum plus pemetrexed, with or without bevacizumab. Preliminary results revealed that the therapy was well tolerated and may be a treatment option for patients responsive to short-term erlotinib treatment (68). In addition, Linsitinib (OSI-906) is an orally bioavailable, dual small-molecule inhibitor of IGF$1 \mathrm{R}$ and insulin receptor (INSR), whose efficacy is currently being tested in clinical studies. A randomized phase II study of the addition of linsitinib to erlotinib in chemotherapy-naive NSCLC patients with positive EGFR mutation demonstrated a detrimental effect that led to inferior efficacy (69). This is not the only clinical trial of linsitinib failing to match the expected results in concord with preclinical data. In a phase II trial, linsitinib maintenance therapy in conjunction with erlotinib showed no difference in progression-free survival (PFS) and overall survival (OS) in NSCLC patients without progression following platinum-based regimen (70). AXL1717 is another small molecular agent which can modulate IGF-1R pathway to develop its antitumor effect. A phase I pilot study designed to definitize maximum tolerated dose (MTD) and recommend phase II dose (RPTD) of AXL1717 adding to gemcitabine $\mathrm{HCl}$ and carboplatin finally established $215 \mathrm{mg}$ BID as MTD and RPTD due to the bone marrow toxicities encountered in this trial (71). Besides, findings from an additional phase II randomized study, in which patients with advanced or metastatic NSCLC were treated with AXL1717 or docetaxel as a single agent, found no statistically significant between two treatment groups. The safety profile in this study concluded that there was a lower frequency of adverse events (AEs), especially neutropenia in the cohort of AXL1717 treatment. Unexpectedly, treatment-related fatal events were noted more commonly in this cohort (12 vs. 5) (72). On the whole, largely undesirable effects encountered in clinical settings emphasize the issue that identification of predictive markers indicative of responses to therapeutic strategies is timely and paramount to improving current therapy in carcinomas.

\section{Blocking Intracellular Signaling Pathways}

With regard to a diverse array of intracellular signaling pathways resulting in EMT, inhibitors of these pathways are in active clinical development. On the basis of compelling preclinical evidence, RAS-RAF-MAPK pathway inhibitors have been put into clinical tests. A recent phase II non-randomized trial of combination BAF and MEK inhibition in patients with BRAF V600E-mutant NSCLC demonstrated a clear clinical benefit of dabrafenib plus trametinib, with a high overall response and manageable toxicity (73). Hampering the PI3k-AKT-mammalian target of rapamycin (mTOR) pathway is another means to prevent or reverse EMT. Pictilisib (GDC-0941), a pan-class PI3k inhibitor, had been reported to possess favorable safety and exert antineoplastic effects in solid tumors, including NSCLC, when administered in monotherapy or combination therapy as well (74-76). Furthermore, a recent phase I study of combination buparlisib (PI3k inhibitor) and radiotherapy in patients with NSCLC showed a well toleration and no dose-limiting toxicity (77). Beyond that, AKT inhibitor MK2206 and mTOR inhibitor everolimus had been tested in the clinic reporting positive results through treatment for cancer $(78,79)$.

As mentioned before, key roles of Notch, Hedgehog and Wnt signal transduction pathways in EMT program and therapeutic resistance are widely embraced. Therefore, various of compounds targeting these pathways are now underway. For Notch cascade, classes of approaches that in clinical pipeline mainly compose of $\gamma$-secretase inhibitors (GSIs) and antibodies against the Notch receptor or ligand (30). At present, considerable clinical trials center on GSIs, which inhibit the generation of NICD to relay transcriptional signals subsequently. So far, a broad range of GSIs, known as PF-03084014, RO4929097, BMS-906024, and LY900009 are under preclinical or early clinical development in solid tumors inclusive of NSCLC (80-83). Besides using GSIs to interfere with the cleavage of Notch receptors, DLL4specific mAbs inhibiting the ligand-receptor interaction reflects another viable option investigated clinically. A phase I FHD study examining enoticumab (REGN421) in eligible patients with advanced solid tumors, to determine the safety, toxicity and RPTD (84). Safety and efficacy of demcizumab in combination with standard chemotherapy was being assessed currently in NSCLC, implying the shorten treatment course in future development (85).

Abnormal activation of the Hedgehog signaling pathway is explicitly implicated in the biological behaviors of cancer, making targeted inhibition of this pathway a clinically useful therapeutic 
strategy. In this respect, a multiple of SMO inhibitors have entered clinical trials (86). As an example, a phase I study evaluating PF-04449913 in advanced solid malignancies showed prolonged stable disease in spite of no objective responses in this trial (87). TAK-441 has been also employed as a potential inhibitor in therapy with the effective outcomes of phase I trial (88). Of note, preliminary data from a study performed that investigated the safety of sonidegib (LDE225) in a total of 45 East Asian patients, suggested an ethnic difference in tolerability between populations (89). As with Notch and Hh pathway, there are intensive researches on targeting Wnt pathway for cancer therapeutics at the stage of preclinical and clinical testing, though no agents have been granted approval, thus far, to target such a pathway $(90,91)$. Recently, a comprehensive body of preclinical evidence that clofazimine specifically inhibits Wnt signaling pathway, without side effect, which possesses potential as a Wnt inhibitor (92).

IL-6 and STAT3 are frequently activated in carcinomas and drive the induction of EMT. An anti-IL-6 mAb, siltuximab, when used clinically to treat advanced solid malignancies, had been proven to be well-tolerated but without objective responses (93). STAT3 is a transcription factor downstream of IL-6 that indirectly induces EMT. A next-generation antisense oligonucleotide (ASO), termed AZD9150, displayed antitumor activity in lung cancer models and a phase I dose-escalation study involving patients with refractory NSCLC (94). Additional clinical trial with OPB51602, a small-molecule STAT3-specific inhibitor, indicated most salient anticancer activity in NSCLC. Given its long half-life and poorer tolerability under continuous dosing, further research is warranted to exploit less frequent dosing schedule (95).

\section{Regulation of miRNAs and Epigenetics}

MiRNAs regulate the EMT process by exciting or undermining EMT-related molecules. Even though a wealth of details is increasingly being recognized between miRNAs and EMT, to date, none of the EMT-associated miRNAs have been applied in clinical utility (44). With expanding knowledge of therapeutic miRNAs in recent years, it is legitimate to say that further work should concentrate on devising miRNA-targeting drugs as a strategy in cancer treatment (96, 97). Apart from the alterations in the genetic landscape, HDACs mediate epigenetic changes during EMT (98). This conceptual advance prompts HDAC inhibitors to translate to the clinic. Of 42 patients with NSCLC and head-and-neck cancer, incorporating an oral pan-HDAC inhibitor, termed as panobinostat (LBH589) with erlotinib potentiated the antitumor effect in a phase I trial (99). In combination with standard erlotinib, romidepsin achieved intriguing clinical activity in populations with NSCLC who did not respond well to EGFR-TKI monotherapy (100).

\section{Targeting CSCs}

Cancer cells gain stem cell-like properties and present a picture of phenotypic diversity, partly brought out by EMT. For this reason, targeting EMT program offers a new ray of hope to eliminate CSCs and improve CSC-based therapy (6). The critical attributes of CSCs - stemness and EMT, contribute to the onset of resistance to therapeutic intervention (101). Cumulatively, treatment strategies operating to direct against CSCs include therapy targeting CSC-dependent signaling pathways, developing anti-EMT approaches and manipulating the tumor microenvironment $(6,51,54)$. First, targeting CSCs via modification of CSC signaling, such as Notch, Hh, Wnt cascade, holds great promise of propelling the clinical update in cancer treatment (32). Second, CSC-targeted therapy can also be accomplished by thwarting EMT-related signaling pathways, given its interconnection with CSC biology (6). Finally, targeting the TME is an alternative possibility in the case of CSCs. As stated, diverse signals within TME can trigger the activation of EMT and entrance cancer cells into the CSC state, thus reducing the vulnerability to targeted agents $(51,54)$. Furthermore, therapeutic functions of miRNAs are coming into prominence for their multifaceted biology to control EMT and EMT-induced CSCs (102). Over the past few years, a renewed interest in immunotherapy for NSCLC treatment conduces to accelerate progress in targeting CSCs with immunotherapeutic methods (103). This knowledge necessitates rigorous clarification of the cellular and molecular mechanisms behind it to produce a durable response to therapy.

\section{CONCLUSIONS}

EGFR as a therapeutic target, its role in patients with EGFRmutant NSCLC is of the essence. Unfortunately, therapeutic susceptibility to EGFR-TKIs is affected by drug resistance (104). With a growing understanding of EMT, the research scope on it is expanding continuously. Most intriguing is how the EMT program gives birth to acquired resistance to EGFR-TKI-based treatment regimes. As argued above, EMT is a kind of highly sophisticated nonlinear dynamic process, which strongly linked with therapeutic responses, stated differently, crosstalk between aforementioned signaling pathways, subjected to genetic and epigenetic modifications, ultimately leading to resistance against anticancer therapy. The complexity and versatility of regulatory mechanisms exceed the expectation, bringing obvious challenges to devise anticancer therapies for clinical use. Another issue is to obtain a solid understanding of EMT-CSC link whereby we can strike at the root of evil (6).

Ongoing studies focusing on EMT make it lie at the heart of drug resistance in oncotherapy. Still, clinical practicable strategies to overcome resistance and improve survival are limited. Given that EMT is a highly regulated process in contextual settings and tightly associated with CSCs, from a therapeutic standpoint, a thorough grasp of mechanism basis is the determinant and first step in the long road fraught with difficulties. Moreover, immunotherapy opens up an attractive area of drug discovery for exploring and treating cancer, while the definition of potential biomarkers that correspond with treatment responses and the development of combinatorial strategies deserve deep concern 
in the future $(105,106)$. The personalized, precise diagnosis and treatment for NSCLC is currently thriving, it is safe to say, therapeutic approaches moving from bench to bedside to ensure optimal efficacy in cancer therapies are on the horizon (107).

\section{REFERENCES}

1. Torre LA, Bray F, Siegel RL, Ferlay J, Lortet-Tieulent J, Jemal A. Global cancer statistics, 2012. CA Cancer J Clin. (2015) 65:87-108. doi: 10.3322/caac.21262

2. Chen Z, Fillmore CM, Hammerman PS, Kim CF, Wong KK. Non-smallcell lung cancers: a heterogeneous set of diseases. Nat Rev Cancer. (2014) 14:535-46. doi: 10.1038/nrc3775

3. Zhou C, Wu YL, Chen G, Feng J, Liu XQ, Wang C, et al. Final overall survival results from a randomised, phase III study of erlotinib versus chemotherapy as first-line treatment of EGFR mutation-positive advanced non-small-cell lung cancer (OPTIMAL, CTONG-0802). Ann Oncol. (2015) 26:1877-83. doi: 10.1093/annonc/mdv276

4. Rosell R, Carcereny E, Gervais R, Vergnenegre A, Massuti B, Felip E, et al. Erlotinib versus standard chemotherapy as first-line treatment for European patients with advanced EGFR mutation-positive non-small-cell lung cancer (EURTAC): a multicentre, open-label, randomised phase 3 trial. Lancet Oncol. (2012) 13:239-46. doi: 10.1016/S1470-2045(11)70393-X

5. Buonato JM, Lazzara MJ. ERK1/2 blockade prevents epithelialmesenchymal transition in lung cancer cells and promotes their sensitivity to EGFR inhibition. Cancer Res. (2014) 74:309-19. doi: 10.1158/0008-5472.CAN-12-4721

6. Shibue T, Weinberg RA. EMT, CSCs, and drug resistance: the mechanistic link and clinical implications. Nat Rev Clin Oncol. (2017) 14:611-29. doi: $10.1038 /$ nrclinonc.2017.44

7. Ye X, Weinberg RA. Epithelial-mesenchymal plasticity: a central regulator of cancer progression. Trends Cell Biol. (2015) 25:675-86. doi: 10.1016/j.tcb.2015.07.012

8. Lamouille S, Xu J, Derynck R. Molecular mechanisms of epithelialmesenchymal transition. Nat Rev Mol Cell Biol. (2014) 15:178-96. doi: $10.1038 / \mathrm{nrm} 3758$

9. Li M, Yang J, Zhou W, Ren Y, Wang X, Chen H, et al. Activation of an AKT/FOXM1/STMN1 pathway drives resistance to tyrosine kinase inhibitors in lung cancer. Br J Cancer. (2017) 117:974-83. doi: 10.1038/bjc. 2017.292

10. Chung JH, Rho JK, Xu X, Lee JS, Yoon HI, Lee CT, et al. Clinical and molecular evidences of epithelial to mesenchymal transition in acquired resistance to EGFR-TKIs. Lung Cancer. (2011) 73:176-82. doi: 10.1016/j.lungcan.2010.11.011

11. Lee AF, Chen MC, Chen CJ, Yang CJ, Huang MS, Liu YP. Reverse epithelialmesenchymal transition contributes to the regain of drug sensitivity in tyrosine kinase inhibitor-resistant non-small cell lung cancer cells. PLoS ONE. (2017) 12:e0180383. doi: 10.1371/journal.pone.0180383

12. Weng CH, Chen LY, Lin YC, Shih JY, Lin YC, Tseng RY, et al. Epithelialmesenchymal transition (EMT) beyond EGFR mutations per se is a common mechanism for acquired resistance to EGFR TKI. Oncogene. (2019) 38:45568. doi: 10.1038/s41388-018-0454-2

13. Yuan $\mathrm{X}, \mathrm{Wu} \mathrm{H}, \mathrm{Han} \mathrm{N}, \mathrm{Xu} \mathrm{H}, \mathrm{Chu} \mathrm{Q}, \mathrm{Yu}$ S, et al. Notch signaling and EMT in non-small cell lung cancer: biological significance and therapeutic application. J Hematol Oncol. (2014) 7:87. doi: 10.1186/s13045-014-0087-z

14. Xie M, He CS, Wei SH, Zhang L. Notch-1 contributes to epidermal growth factor receptor tyrosine kinase inhibitor acquired resistance in non-small cell lung cancer in vitro and in vivo. Eur J Cancer. (2013) 49:3559-72. doi: 10.1016/j.ejca.2013.07.007

15. Nieto MA, Huang RY, Jackson RA, Thiery JP. EMT: 2016. Cell. (2016) 166:21-45. doi: 10.1016/j.cell.2016.06.028

16. Gupta S, Maitra A. EMT: matter of life or death? Cell. (2016) 164:840-2. doi: 10.1016/j.cell.2016.02.024

17. David CJ, Huang YH, Chen M, Su J, Zou Y, Bardeesy N, et al. TGFbeta tumor suppression through a lethal EMT. Cell. (2016) 164:1015-30. doi: 10.1016/j.cell.2016.01.009

\section{AUTHOR CONTRIBUTIONS}

$\mathrm{XN}$ conceived the project direction. XZ and LC inquired and collated all the literature. LC and LL read all the literature and wrote the manuscript. XN drawn the mechanism diagram.

18. Soucheray M, Capelletti M, Pulido I, Kuang Y, Paweletz CP, Becker JH, et al. Intratumoral heterogeneity in EGFR-mutant NSCLC results in divergent resistance mechanisms in response to EGFR tyrosine kinase inhibition. Cancer Res. (2015) 75:4372-83. doi: 10.1158/0008-5472.CAN-15-0377

19. Yoshida T, Song L, Bai Y, Kinose F, Li J, Ohaegbulam KC, et al. ZEB1 mediates acquired resistance to the epidermal growth factor receptor-tyrosine kinase inhibitors in non-small cell lung cancer. PLoS ONE. (2016) 11:e0147344. doi: 10.1371/journal.pone.0147344

20. Lee MK, Pardoux C, Hall MC, Lee PS, Warburton D, Qing J, et al. TGF-beta activates Erk MAP kinase signalling through direct phosphorylation of ShcA. EMBO J. (2007) 26:3957-67. doi: 10.1038/sj.emboj.7601818

21. Derynck R, Muthusamy BP, Saeteurn KY. Signaling pathway cooperation in TGF-beta-induced epithelial-mesenchymal transition. Curr Opin Cell Biol. (2014) 31:56-66. doi: 10.1016/j.ceb.2014.09.001

22. Deskin B, Lasky J, Zhuang Y, Shan B. Requirement of HDAC6 for activation of Notch1 by TGF-beta1. Sci Rep. (2016) 6:31086. doi: 10.1038/srep31086

23. Wang Z, Tang F, Hu P, Wang Y, Gong J, Sun S, et al. HDAC6 promotes cell proliferation and confers resistance to gefitinib in lung adenocarcinoma. Oncol Rep. (2016) 36:589-97. doi: 10.3892/or.2016.4811

24. Li H, Batth IS, Qu X, Xu L, Song N, Wang R, et al. IGF-IR signaling in epithelial to mesenchymal transition and targeting IGF-IR therapy: overview and new insights. Mol Cancer. (2017) 16:6. doi: 10.1186/s12943-016-0576-5

25. Denduluri SK, Idowu O, Wang Z, Liao Z, Yan Z, Mohammed MK, et al. Insulin-like growth factor (IGF) signaling in tumorigenesis and the development of cancer drug resistance. Genes Dis. (2015) 2:13-25. doi: 10.1016/j.gendis.2014.10.004

26. Zhou J, Wang J, Zeng Y, Zhang X, Hu Q, Zheng J, et al. Implication of epithelial-mesenchymal transition in IGF1R-induced resistance to EGFRTKIs in advanced non-small cell lung cancer. Oncotarget. (2015) 6:44332-45. doi: 10.18632/oncotarget.6293

27. Yeo CD, Park KH, Park CK, Lee SH, Kim SJ, Yoon HK, et al. Expression of insulin-like growth factor 1 receptor (IGF-1R) predicts poor responses to epidermal growth factor receptor (EGFR) tyrosine kinase inhibitors in nonsmall cell lung cancer patients harboring activating EGFR mutations. Lung Cancer. (2015) 87:311-7. doi: 10.1016/j.lungcan.2015.01.004

28. Hussmann D, Madsen AT, Jakobsen KR, Luo Y, Sorensen BS, Nielsen AL. IGF1R depletion facilitates MET-amplification as mechanism of acquired resistance to erlotinib in HCC827 NSCLC cells. Oncotarget. (2017) 8:3330015. doi: 10.18632/oncotarget.16350

29. Yuan X, Wu H, Xu H, Xiong H, Chu Q, Yu S, et al. Notch signaling: an emerging therapeutic target for cancer treatment. Cancer Lett. (2015) 369:20-7. doi: 10.1016/j.canlet.2015.07.048

30. Andersson ER, Lendahl U. Therapeutic modulation of Notch signalling-are we there yet? Nat Rev Drug Discov. (2014) 13:357-78. doi: 10.1038/nrd4252

31. Zhang T, Guo L, Creighton CJ, Lu Q, Gibbons DL, Yi ES, et al. A genetic cell context-dependent role for ZEB1 in lung cancer. Nat Commun. (2016) 7:12231. doi: 10.1038/ncomms12231

32. Takebe N, Miele L, Harris PJ, Jeong W, Bando H, Kahn M, et al. Targeting Notch, Hedgehog, and Wnt pathways in cancer stem cells: clinical update. Nat Rev Clin Oncol. (2015) 12:445-64. doi: 10.1038/nrclinonc.2015.61

33. Briscoe J, Thérond PP. The mechanisms of Hedgehog signalling and its roles in development and disease. Nat Rev Mol Cell Biol. (2013) 14:416-29. doi: $10.1038 / \mathrm{nrm} 3598$

34. Della Corte CM, Bellevicine C, Vicidomini G, Vitagliano D, Malapelle U, Accardo M, et al. SMO gene amplification and activation of the hedgehog pathway as novel mechanisms of resistance to anti-epidermal growth factor receptor drugs in human lung cancer. Clin Cancer Res. (2015) 21:4686-97. doi: 10.1158/1078-0432.CCR-14-3319

35. Bai XY, Zhang XC, Yang SQ, An SJ, Chen ZH, Su J, et al. Blockade of hedgehog signaling synergistically increases sensitivity to epidermal growth 
factor receptor tyrosine kinase inhibitors in non-small-cell lung cancer cell lines. PLoS ONE. (2016) 11:e0149370. doi: 10.1371/journal.pone.0149370

36. Della Corte CM, Malapelle U, Vigliar E, Pepe F, Troncone G, Ciaramella V, et al. Efficacy of continuous EGFR-inhibition and role of Hedgehog in EGFR acquired resistance in human lung cancer cells with activating mutation of EGFR. Oncotarget. (2017) 8:23020-32. doi: 10.18632/oncotarget.15479

37. Li D, Zhang L, Zhou J, Chen H. Cigarette smoke extract exposure induces EGFR-TKI resistance in EGFR-mutated NSCLC via mediating Src activation and EMT. Lung Cancer. (2016) 93:35-42. doi: 10.1016/j.lungcan.2015.12.007

38. Liu M, Zhou C, Zheng J. Cigarette smoking impairs the response of EGFRTKIs therapy in lung adenocarcinoma patients by promoting EGFR signaling and epithelial-mesenchymal transition. Am J Transl Res. (2015) 7:2026-35.

39. Li L, Han R, Xiao H, Lin C, Wang Y, Liu H, et al. Metformin sensitizes EGFRTKI-resistant human lung cancer cells in vitro and in vivo through inhibition of IL-6 signaling and EMT reversal. Clin Cancer Res. (2014) 20:2714-26. doi: 10.1158/1078-0432.CCR-13-2613

40. Shien K, Papadimitrakopoulou VA, Ruder D, Behrens C, Shen L, Kalhor N, et al. JAK1/STAT3 activation through a proinflammatory cytokine pathway leads to resistance to molecularly targeted therapy in non-small cell lung cancer. Mol Cancer Ther. (2017) 16:2234-45. doi: 10.1158/1535-7163.MCT-17-0148

41. Kim G, An HJ, Lee MJ, Song JY, Jeong JY, Lee JH, et al. Hsa-miR1246 and hsa-miR-1290 are associated with stemness and invasiveness of non-small cell lung cancer. Lung Cancer. (2016) 91:15-22. doi: 10.1016/j.lungcan.2015.11.013

42. Zeng Y, Zhu J, Shen D, Qin H, Lei Z, Li W, et al. Repression of Smad4 by miR205 moderates TGF-beta-induced epithelial-mesenchymal transition in A549 cell lines. Int J Oncol. (2016) 49:700-8. doi: 10.3892/ijo.2016.3547

43. Yamashita R, Sato M, Kakumu T, Hase T, Yogo N, Maruyama E, et al. Growth inhibitory effects of miR-221 and miR-222 in non-small cell lung cancer cells. Cancer Med. (2015) 4:551-64. doi: 10.1002/cam4.412

44. Tang J, Li Y, Wang J, Wen Z, Lai M, Zhang H. Molecular mechanisms of microRNAs in regulating epithelial-mesenchymal transitions in human cancers. Cancer Lett. (2016) 371:301-13. doi: 10.1016/j.canlet.2015. 11.043

45. Chen Y, Lu L, Feng B, Han S, Cui S, Chu X, et al. Non-coding RNAs as emerging regulators of epithelial to mesenchymal transition in non-small cell lung cancer. Oncotarget. (2017) 8:36787-99. doi: 10.18632/oncotarget.16375

46. Narita M, Shimura E, Nagasawa A, Aiuchi T, Suda Y, Hamada Y, et al. Chronic treatment of non-small-cell lung cancer cells with gefitinib leads to an epigenetic loss of epithelial properties associated with reductions in microRNA-155 and-200c. PLoS ONE. (2017) 12:e0172115. doi: 10.1371/journal.pone.0172115

47. Mutlu M, Raza U, Saatci Ö, Eyüpoğlu E, Yurdusev E, Şahin Ö. miR-200c: a versatile watchdog in cancer progression, EMT, and drug resistance. J Mol Med (Berl). (2016) 94:629-44. doi: 10.1007/s00109-016$1420-5$

48. Sato H, Shien K, Tomida S, Okayasu K, Suzawa K, Hashida S, et al. Targeting the miR-200c/LIN28B axis in acquired EGFR-TKI resistance nonsmall cell lung cancer cells harboring EMT features. Sci Rep. (2017) 7:40847. doi: 10.1038/srep40847

49. Liu YN, Chang TH, Tsai MF, Wu SG, Tsai TH, Chen HY, et al. IL-8 confers resistance to EGFR inhibitors by inducing stem cell properties in lung cancer. Oncotarget. (2015) 6: 10415-31. doi: 10.18632/oncotarget.3389

50. de Aberasturi AL, Redrado M, Villalba M, Larzabal L, Pajares MJ, Garcia J, et al. TMPRSS4 induces cancer stem cell-like properties in lung cancer cells and correlates with ALDH expression in NSCLC patients. Cancer Lett. (2016) 370:165-76. doi: 10.1016/j.canlet.2015.10.012

51. Pattabiraman DR, Weinberg RA. Tackling the cancer stem cells-what challenges do they pose? Nat Rev Drug Discov. (2014) 13:497-512. doi: $10.1038 / \mathrm{nrd} 4253$

52. Hashida S, Yamamoto H, Shien K, Miyoshi Y, Ohtsuka T, Suzawa K, et al. Acquisition of cancer stem cell-like properties in non-small cell lung cancer with acquired resistance to afatinib. Cancer Sci. (2015) 106:1377-84. doi: $10.1111 /$ cas. 12749

53. Yin H, Wang Y, Chen W, Zhong S, Liu Z, Zhao J. Drug-resistant CXCR4positive cells have the molecular characteristics of EMT in NSCLC. Gene. (2016) 594:23-9. doi: 10.1016/j.gene.2016.08.043
54. Pützer BM, Solanki M, Herchenröder O. Advances in cancer stem cell targeting: how to strike the evil at its root. Adv Drug Deliv Rev. (2017) 120:89-107. doi: 10.1016/j.addr.2017.07.013

55. Sato R, Semba T, Saya H, Arima Y. Concise review: stem cells and epithelialmesenchymal transition in cancer: biological implications and therapeutic targets. Stem Cells. (2016) 34:1997-2007. doi: 10.1002/stem.2406

56. Lin JJ, Shaw AT. Resisting resistance: targeted therapies in lung cancer. Trends Cancer. (2016) 2:350-64. doi: 10.1016/j.trecan.2016.05.010

57. Marcucci F, Stassi G, De Maria R. Epithelial-mesenchymal transition: a new target in anticancer drug discovery. Nat Rev Drug Discov. (2016) 15:311-25. doi: 10.1038/nrd.2015.13

58. Colak S, Ten Dijke P. Targeting TGF-beta signaling in cancer. Trends Cancer. (2017) 3:56-71. doi: 10.1016/j.trecan.2016.11.008

59. Herbertz S, Sawyer JS, Stauber AJ, Gueorguieva I, Driscoll KE, Estrem ST, et al. Clinical development of galunisertib (LY2157299 monohydrate), a small molecule inhibitor of transforming growth factor-beta signaling pathway. Drug Des Devel Ther. (2015) 9:4479-99. doi: 10.2147/DDDT.S86621

60. Rodón J, Carducci M, Sepulveda-Sánchez JM, Azaro A, Calvo E, Seoane J, et al. Pharmacokinetic, pharmacodynamic and biomarker evaluation of transforming growth factor-beta receptor I kinase inhibitor, galunisertib, in phase 1 study in patients with advanced cancer. Invest New Drugs. (2015) 33:357-70. doi: 10.1007/s10637-014-0192-4

61. Fujiwara Y, Nokihara H, Yamada Y, Yamamoto N, Sunami K, Utsumi H, et al. Phase 1 study of galunisertib, a TGF-beta receptor I kinase inhibitor, in Japanese patients with advanced solid tumors. Cancer Chemother Pharmacol. (2015) 76:1143-52. doi: 10.1007/s00280-015-2895-4

62. Kelley RK, Gane E, Assenat E, Siebler J, Galle PR, Merle P, et al. A phase 2 study of galunisertib (TGF-betal receptor type i inhibitor) and sorafenib in patients with advanced hepatocellular carcinoma. Clin Transl Gastroenterol. (2019) 10:e00056. doi: 10.14309/ctg.0000000000000056

63. Kovacs RJ, Maldonado G, Azaro A, Fernández MS, Romero FL, SepulvedaSánchez JM, et al. Cardiac safety of TGF-beta receptor I kinase inhibitor LY2157299 monohydrate in cancer patients in a first-in-human dose study. Cardiovasc Toxicol. (2015) 15:309-23. doi: 10.1007/s12012-014-9297-4

64. Giaccone G, Bazhenova LA, Nemunaitis J, Tan M, Juhász E, Ramlau R, et al. A phase III study of belagenpumatucel-L, an allogeneic tumour cell vaccine, as maintenance therapy for non-small cell lung cancer. Eur J Cancer. (2015) 51:2321-9. doi: 10.1016/j.ejca.2015.07.035

65. Iams WT, Lovly CM. Molecular pathways: clinical applications and future direction of insulin-like growth factor-1 receptor pathway blockade. Clin Cancer Res. (2015) 21:4270-7. doi: 10.1158/1078-0432.CCR-14-2518

66. Scagliotti GV, Bondarenko I, Blackhall F, Barlesi F, Hsia TC, Jassem J, et al. Randomized, phase III trial of figitumumab in combination with erlotinib versus erlotinib alone in patients with nonadenocarcinoma nonsmall-cell lung cancer. Ann Oncol. (2015) 26:497-504. doi: 10.1093/annonc/mdu517

67. Langer CJ, Novello S, Park K, Krzakowski M, Karp DD, Mok T, et al. Randomized, phase III trial of first-line figitumumab in combination with paclitaxel and carboplatin versus paclitaxel and carboplatin alone in patients with advanced non-small-cell lung cancer. J Clin Oncol. (2014) 32:2059-66. doi: 10.1200/JCO.2013.54.4932

68. Tani T, Naoki K, Yasuda H, Arai D, Ishioka K, Ohgino K, et al. A phase II trial of induction of erlotinib followed by cytotoxic chemotherapy for EGFR mutation-positive non-squamous non-small cell lung cancer patients. Cancer Chemother Pharmacol. (2019). doi: 10.1007/s00280-019-03934-y. [Epub ahead of print].

69. Leighl NB, Rizvi NA, de Lima LG, Arpornwirat W, Rudin CM, Chiappori AA, et al. Phase 2 study of erlotinib in combination with linsitinib (OSI906) or placebo in chemotherapy-naive patients with non-small-cell lung cancer and activating epidermal growth factor receptor mutations. Clin Lung Cancer. (2017) 18:34-42.e2. doi: 10.1016/j.cllc.2016.07.007

70. Ciuleanu TE, Ahmed S, Kim JH, Mezger J, Park K, Thomas M, et al. Randomised Phase 2 study of maintenance linsitinib (OSI-906) in combination with erlotinib compared with placebo plus erlotinib after platinum-based chemotherapy in patients with advanced non-small cell lung cancer. Br J Cancer. (2017) 117:757-66. doi: 10.1038/bjc.2017.226

71. Holgersson G, Bergstrom S, Harmenberg J, Ringbom M, Klockare M, Jerling $M$, et al. A phase I pilot study of the insulin-like growth 
factor 1 receptor pathway modulator AXL1717 in combination with gemcitabine $\mathrm{HCl}$ and carboplatin in previously untreated, locally advanced, or metastatic non-small cell lung cancer. Med Oncol. (2015) 32:129. doi: 10.1007/s12032-015-0578-y

72. Bergqvist M, Holgersson G, Bondarenko I, Grechanaya E, Maximovich A, Andor G, et al. Phase II randomized study of the IGF-1R pathway modulator AXL1717 compared to docetaxel in patients with previously treated, locally advanced or metastatic non-small cell lung cancer. Acta Oncol. (2017) 56:441-7. doi: 10.1080/0284186X.2016.1253866

73. Planchard D, Smit EF, Groen HJM, Mazieres J, Besse B, Helland $\AA$, et al. Dabrafenib plus trametinib in patients with previously untreated BRAF(V600E)-mutant metastatic non-small-cell lung cancer: an open-label, phase 2 trial. Lancet Oncol. (2017) 18:1307-16. doi: 10.1016/S1470-2045(17)30679-4

74. Soria JC, Adjei AA, Bahleda R, Besse B, Ferte C, Planchard D, et al. A phase IB dose-escalation study of the safety and pharmacokinetics of pictilisib in combination with either paclitaxel and carboplatin (with or without bevacizumab) or pemetrexed and cisplatin (with or without bevacizumab) in patients with advanced non-small cell lung cancer. Eur J Cancer. (2017) 86:186-96. doi: 10.1016/j.ejca.2017.08.027

75. Yamamoto N, Fujiwara Y, Tamura K, Kondo S, Iwasa S, Tanabe Y, et al. Phase Ia/Ib study of the pan-class I PI3K inhibitor pictilisib (GDC-0941) administered as a single agent in Japanese patients with solid tumors and in combination in Japanese patients with non-squamous non-small cell lung cancer. Invest New Drugs. (2017) 35:37-46. doi: 10.1007/s10637-016-0382-3

76. Sarker D, Ang JE, Baird R, Kristeleit R, Shah K, Moreno V, et al. First-in-human phase I study of pictilisib (GDC-0941), a potent panclass I phosphatidylinositol-3-kinase (PI3K) inhibitor, in patients with advanced solid tumors. Clin Cancer Res. (2015) 21:77-86. doi: 10.1158/1078-0432.CCR-14-0947

77. McGowan DR, Skwarski M, Bradley KM, Campo L, Fenwick JD, Gleeson FV, et al. Buparlisib with thoracic radiotherapy and its effect on tumour hypoxia: a phase I study in patients with advanced non-small cell lung carcinoma. Eur J Cancer. (2019) 113:87-95. doi: 10.1016/j.ejca.2019.03.015

78. Molife LR, Yan L, Vitfell-Rasmussen J, Zernhelt AM, Sullivan DM, Cassier PA, et al. Phase 1 trial of the oral AKT inhibitor MK-2206 plus carboplatin/paclitaxel, docetaxel, or erlotinib in patients with advanced solid tumors. J Hematol Oncol. (2014) 7:1. doi: 10.1186/1756-8722-7-1

79. Owonikoko TK, Ramalingam SS, Miller DL, Force SD, Sica GL, Mendel J, et al. A translational, pharmacodynamic, and pharmacokinetic phase IB clinical study of everolimus in resectable non-small cell lung cancer. Clin Cancer Res. (2015) 21:1859-68. doi: 10.1158/1078-0432.CCR-14-1998

80. Pant S, Jones SF, Kurkjian CD, Infante JR, Moore KN, Burris HA, et al. A first-in-human phase I study of the oral Notch inhibitor, LY900009, in patients with advanced cancer. Eur J Cancer. (2016) 56:1-9. doi: 10.1016/j.ejca.2015.11.021

81. Gavai AV, Quesnelle C, Norris D, Han WC, Gill P, Shan W, et al. Discovery of clinical candidate BMS-906024: a potent pan-notch inhibitor for the treatment of leukemia and solid tumors. ACS Med Chem Lett. (2015) 6:523-7. doi: 10.1021/acsmedchemlett.5b00001

82. LoConte NK, Razak AR, Ivy P, Tevaarwerk A, Leverence R, Kolesar J, et al. A multicenter phase 1 study of gamma -secretase inhibitor RO4929097 in combination with capecitabine in refractory solid tumors. Invest New Drugs. (2015) 33:169-76. doi: 10.1007/s10637-014-0166-6

83. Messersmith WA, Shapiro GI, Cleary JM, Jimeno A, Dasari A, Huang B, et al. A Phase I, dose-finding study in patients with advanced solid malignancies of the oral gamma-secretase inhibitor PF-03084014. Clin Cancer Res. (2015) 21:60-7. doi: 10.1158/1078-0432.CCR-14-0607

84. Chiorean EG, LoRusso P, Strother RM, Diamond JR, Younger A, Messersmith WA, et al. A phase I first-in-human study of enoticumab (REGN421), a fully human delta-like ligand 4 (Dll4) monoclonal antibody in patients with advanced solid tumors. Clin Cancer Res. (2015) 21:2695-703. doi: 10.1158/1078-0432.CCR-14-2797

85. McKeage MJ, Kotasek D, Markman B, Hidalgo M, Millward MJ, Jameson $\mathrm{MB}$, et al. Phase IB trial of the anti-cancer stem cell DLL4-binding agent demcizumab with pemetrexed and carboplatin as first-line treatment of metastatic non-squamous NSCLC. Target Oncol. (2018) 13:89-98. doi: $10.1007 /$ s11523-017-0543-0
86. Xin M, Ji X, De La Cruz LK, Thareja S, Wang B. Strategies to target the Hedgehog signaling pathway for cancer therapy. Med Res Rev. (2018) 38:870-913. doi: $10.1002 /$ med.21482

87. Wagner AJ, Messersmith WA, Shaik MN, Li S, Zheng X, McLachlan KR, et al. A phase I study of PF-04449913, an oral hedgehog inhibitor, in patients with advanced solid tumors. Clin Cancer Res. (2015) 21:1044-51. doi: 10.1158/1078-0432.CCR-14-1116

88. Goldman J, Eckhardt SG, Borad MJ, Curtis KK, Hidalgo M, Calvo E, et al. Phase I dose-escalation trial of the oral investigational Hedgehog signaling pathway inhibitor TAK-441 in patients with advanced solid tumors. Clin Cancer Res. (2015) 21:1002-9. doi: 10.1158/1078-0432.CCR-14-1234

89. Minami H, Ando Y, Ma BB, Hsiang Lee J, Momota H, Fujiwara Y, et al. Phase I, multicenter, open-label, dose-escalation study of sonidegib in Asian patients with advanced solid tumors. Cancer Sci. (2016) 107:1477-83. doi: $10.1111 /$ cas. 13022

90. Krishnamurthy N, Kurzrock R. Targeting the Wnt/beta-catenin pathway in cancer: update on effectors and inhibitors. Cancer Treat Rev. (2018) 62:50-60. doi: 10.1016/j.ctrv.2017.11.002

91. Tai D, Wells K, Arcaroli J, Vanderbilt C, Aisner DL, Messersmith WA, et al. Targeting the WNT signaling pathway in cancer therapeutics. Oncologist. (2015) 20:1189-98. doi: 10.1634/theoncologist.2015-0057

92. Ahmed K, Koval A, Xu J, Bodmer A, Katanaev VL. Towards the first targeted therapy for triple-negative breast cancer: repositioning of clofazimine as a chemotherapy-compatible selective Wnt pathway inhibitor. Cancer Lett. (2019) 449:45-55. doi: 10.1016/j.canlet.2019.02.018

93. Angevin E, Tabernero J, Elez E, Cohen SJ, Bahleda R, van Laethem JL, et al. A phase I/II, multiple-dose, dose-escalation study of siltuximab, an antiinterleukin-6 monoclonal antibody, in patients with advanced solid tumors. Clin Cancer Res. (2014) 20:2192-204. doi: 10.1158/1078-0432.CCR-13-2200

94. Hong D, Kurzrock R, Kim Y, Woessner R, Younes A, Nemunaitis J, et al. AZD9150, a next-generation antisense oligonucleotide inhibitor of STAT3 with early evidence of clinical activity in lymphoma and lung cancer. Sci Transl Med. (2015) 7:314ra185. doi: 10.1126/scitranslmed. aac5272

95. Wong AL, Soo RA, Tan DS, Lee SC, Lim JS, Marban PC, et al. Phase I and biomarker study of OPB-51602, a novel signal transducer and activator of transcription (STAT) 3 inhibitor, in patients with refractory solid malignancies. Ann Oncol. (2015) 26:998-1005. doi: 10.1093/annonc/mdv026

96. Chakraborty C, Sharma AR, Sharma G, Doss CGP, Lee SS. Therapeutic miRNA and siRNA: moving from bench to clinic as next generation medicine. Mol Ther Nucleic Acids. (2017) 8:132-43. doi: 10.1016/j.omtn.2017.06.005

97. Ling H, Fabbri M, Calin GA. MicroRNAs and other non-coding RNAs as targets for anticancer drug development. Nat Rev Drug Discov. (2013) 12:847-65. doi: 10.1038/nrd4140

98. Serrano-Gomez SJ, Maziveyi M, Alahari SK. Regulation of epithelialmesenchymal transition through epigenetic and post-translational modifications. Mol Cancer. (2016) 15:18. doi: 10.1186/s12943-016-0502-x

99. Gray JE, Haura E, Chiappori A, Tanvetyanon T, Williams CC, PinderSchenck M, et al. A phase I, pharmacokinetic, and pharmacodynamic study of panobinostat, an HDAC inhibitor, combined with erlotinib in patients with advanced aerodigestive tract tumors. Clin Cancer Res. (2014) 20:164455. doi: 10.1158/1078-0432.CCR-13-2235

100. Gerber DE, Boothman DA, Fattah FJ, Dong Y, Zhu H, Skelton RA, et al. Phase 1 study of romidepsin plus erlotinib in advanced non-small cell lung cancer. Lung Cancer. (2015) 90:534-41. doi: 10.1016/j.lungcan.2015.10.008

101. Rosa R, D’Amato V, De Placido S, Bianco R. Approaches for targeting cancer stem cells drug resistance. Expert Opin Drug Discov. (2016) 11:1201-12. doi: 10.1080/17460441.2016.1243525

102. Garg M. Targeting microRNAs in epithelial-to-mesenchymal transitioninduced cancer stem cells: therapeutic approaches in cancer. Expert Opin Ther Targets. (2015) 19:285-97. doi: 10.1517/14728222.2014.975794

103. Pan Q, Li Q, Liu S, Ning N, Zhang X, Xu Y, et al. Concise review: targeting cancer stem cells using immunologic approaches. Stem Cells. (2015) 33:208592. doi: 10.1002/stem.2039

104. Ke EE, Wu YL. EGFR as a pharmacological target in EGFR-mutant nonsmall-cell lung cancer: where do we stand now? Trends Pharmacol Sci. (2016) 37:887-903. doi: 10.1016/j.tips.2016.09.003 
105. Rizvi NA, Hellmann MD, Snyder A, Kvistborg P, Makarov V, Havel JJ, et al. Mutational landscape determines sensitivity to PD-1 blockade in non-small cell lung cancer. Science. (2015) 348:124-8. doi: 10.1126/science. aaa 1348

106. Anagnostou VK, Brahmer JR. Cancer immunotherapy: a future paradigm shift in the treatment of non-small cell lung cancer. Clin Cancer Res. (2015) 21:976-84. doi: 10.1158/1078-0432.CCR-14-1187

107. Reck M, Rabe KF. Precision diagnosis and treatment for advanced non-small-cell lung cancer. $N$ Engl J Med. (2017) 377:849-61. doi: 10.1056/NEJMra1703413
Conflict of Interest: The authors declare that the research was conducted in the absence of any commercial or financial relationships that could be construed as a potential conflict of interest.

Copyright (c) 2019 Zhu, Chen, Liu and Niu. This is an open-access article distributed under the terms of the Creative Commons Attribution License (CC BY). The use, distribution or reproduction in other forums is permitted, provided the original author(s) and the copyright owner(s) are credited and that the original publication in this journal is cited, in accordance with accepted academic practice. No use, distribution or reproduction is permitted which does not comply with these terms. 